\title{
Abundances and chemical stratification analysis in the atmosphere of Cr-type Ap star HD 204411 ^
}

\author{
T. Ryabchikova ${ }^{1,2}$, F. Leone ${ }^{3}$, and O. Kochukhov ${ }^{2, \star \star}$ \\ ${ }^{1}$ Institute for Astronomy, Russian Academy of Sciences, Pyatnitskaya 48, 119017 Moscow, Russia \\ e-mail: ryabchik@inasan.rssi.ru \\ ${ }^{2}$ Institute for Astronomy, University of Vienna, Türkenschanzstraße 17, 1180 Vienna, Austria \\ e-mail: last_name@astro.univie.ac.at; oleg@astro.uu.se \\ 3 INAF - Osservatorio Astrofisico di Catania, Via S. Sofia 78, 95123 Catania, Italy \\ e-mail: fleone@ct.astro.it
}

Received 12 September 2004 / Accepted 31 March 2005

\begin{abstract}
We present results of an abundance and stratification analysis of the weakly magnetic chemically peculiar star HD 204411 based on the echelle spectrum obtained with the high resolution spectrograph at the 3.55-m Telescopio Nazionale Galileo at the Observatorio del Roque de los Muchachos (La Palma, Spain). Atmospheric parameters obtained from the spectroscopy and spectrophotometry together with the Hipparcos parallax show that this star has already left the Main Sequence band. The upper limit for the surface magnetic field derived from the differential broadening of the spectral lines with different magnetic sensitivity is $750 \mathrm{G}$, which agrees with the recent detection of the weak effective magnetic field in this star. The best fit to the observed spectral line profiles was obtained with a combination of the rotational velocity $v_{\mathrm{e}} \sin i=5.4 \mathrm{~km} \mathrm{~s}^{-1}$ and the radial-tangential macroturbulence of $4.8 \mathrm{~km} \mathrm{~s}^{-1}$. The average abundances of HD 204411 are typical for an Ap star of the Cr-type: $\mathrm{C}$ and $\mathrm{O}$ are deficient, $\mathrm{Cr}$ and $\mathrm{Fe}$ are strongly overabundant. $\mathrm{Sr}, \mathrm{Y}, \mathrm{Zr}$ and the rare-earths, which usually have large overabundances in cool Ap stars with strong magnetic fields, are either normal ( $\mathrm{Y}, \mathrm{Ce}$ ) or only +0.5 dex overabundant in the weakly magnetic star HD 204411.

The chemical stratification analysis was performed for 5 elements, $\mathrm{Mg}, \mathrm{Si}, \mathrm{Ca}, \mathrm{Cr}$ and $\mathrm{Fe} . \mathrm{Si}, \mathrm{Ca}$ and $\mathrm{Fe}$ show a tendency to be concentrated below $\log \tau_{5000}=-1$, while for $\mathrm{Mg}$ we found marginal evidence for concentration in the upper atmosphere. This behaviour of Mg may be an artifact caused by the limited sample of spectral lines and poor atomic data available for the Mg II lines used in our analysis. Chromium, the most anomalous Fe-peak element, does not show significant abundance gradients in the line-forming region.
\end{abstract}

Key words. stars: atmospheres - stars: chemically peculiar - stars: abundances - stars: individual: HD 204411

\section{Introduction}

HD 204411 (=HR 8216) was classified as an Ap star of the CrEu-type by Morgan (1932). However, the first abundance analysis of this star (Sargent et al. 1969) did not reveal the Eu overabundance typical of this type of peculiar star. They reported only an upper limit of +1.0 dex relative to the Sun. Cowley \& Henry (1979) placed HD 204411 in the subgroup of Ap stars where "iron group spectra are usually strong, but lanthanide spectra may be weaker than in "normal" Ap stars".

^ Based on observations made with the Italian Telescopio Nazionale Galileo (TNG) operated on the island of La Palma by the Centro Galileo Galilei of the INAF (Istituto Nazionale di Astrofisica) at the Spanish Observatorio del Roque de los Muchachos of the Instituto de Astrofisica de Canarias and on spectral data retrieved from the ELODIE archive at Observatoire de Haute-Provence (OHP).

$\star \star$ Present address: Department of Astronomy and Space Physics, Uppsala University, Box 515, 75120 Uppsala, Sweden.
The most recent abundance analysis of HD 204411 based on the Reticon spectra (Caliskan \& Adelman 1995 - CA) fully supported this classification. Except for Pr, they found less than 1 dex overabundance for the other rare-earth elements (REE). $\mathrm{Ti}, \mathrm{Cr}, \mathrm{Fe}$ and $\mathrm{Co}$ are overabundant by $0.5 \mathrm{dex}$ or more. The largest anomaly in HD 204411 is overabundance of Cr by $1.5 \mathrm{dex}$. The atmospheric parameters, $T_{\text {eff }}=8400 \mathrm{~K}$ and especially the unusually low surface gravity $\log g=3.3$, derived by Caliskan \& Adelman (1995) from the optical region spectrophotometry and the $\mathrm{H} \gamma$ line profile, suggest that the star is evolved.

The magnetic field of HD 204411 should be weak as follows from the only estimate for the surface magnetic field of $0.5 \mathrm{kG}$ (Preston 1971). This estimate was based on the difference of half-widths of the magnetically sensitive and insensitive lines. This difference is zero for HD 204411 and the $0.5 \mathrm{kG}$ estimate strongly depends on the calibration used by Preston. However, the presence of the weak magnetic field in 
HD 204411 was supported by Johnson (2004) who measured a longitudinal component of the magnetic field (effective magnetic field), $B_{\mathrm{e}}=30 \pm 11 \mathrm{G}$. A weak or zero magnetic field is not typical of the CrEu stars.

HD 204411 was suggested by Preston (1970) as a candidate very long period variable. Indeed, photometry over a few years did not show significant variations (Adelman et al. 1994). However, from the analysis of the $u v b y$ photometry obtained in 1991-2001, Adelman (2003) found a change in $b$ (-0.016 mag) and $v(-0.010 \mathrm{mag})$. The possible period of the photometric variations is larger than 20 years. Considering this period as the rotational one, we expect to see extremely sharp lines in the spectrum of HD 204411. However, the existing measurements provide rather high rotational velocities: $15 \mathrm{~km} \mathrm{~s}^{-1}$ (Abt \& Morrell 1995), $23 \mathrm{~km} \mathrm{~s}^{-1}$ (Royer et al. 2002), whereas CA were able to determine only the upper limit, $v_{\mathrm{e}} \sin i<5 \mathrm{~km} \mathrm{~s}^{-1}$.

The chemical analysis results presented by $\mathrm{CA}$ provided the mean abundances without error estimates (except for $\mathrm{Ti}, \mathrm{Cr}$, $\mathrm{Fe}$ ) and without a separate assessment of the neutral and ionized species. They also did not derive abundances for the light elements, such as CNO. Moreover, a rather large standard deviation of the $\mathrm{Ti}, \mathrm{Cr}$ and $\mathrm{Fe}$ abundances for this slowly rotating, practically non-magnetic star suggests the possible influence of vertical chemical inhomogeneities, which were recently found in cool Ap stars (Wade et al. 2001; Ryabchikova et al. 2002).

The main goal of the present paper is to carry out a more detailed spectroscopic and abundance analysis to derive an abundance distribution for different chemical elements through the atmosphere. These data provide crucial observational constraints for the theoretical modelling of the radiative diffusion in the atmospheres of Ap stars. For this purpose we obtained a new high-resolution, high signal-to-noise spectrum of HD 204411. Observations and data reduction are described in Sect. 2. Atmospheric parameters, evolutionary status, magnetic field and rotational velocity determination are presented in Sect. 3. Results of the abundance analysis in the homogeneous atmosphere approximation are given in Sect. 4, and our stratification analysis is described in Sect. 5.

\section{Observations and spectrum reduction}

An echelle spectrum $(R=164000)$ of HD 204411 was obtained on August 1, 2001 (HJD = 2452 122.572) with the high resolution spectrograph $(S A R G)$ at the 3.55-m Telescopio Nazionale Galileo (TNG) at the Observatorio del Roque de los Muchachos (La Palma, Spain). Using the yellow grism we selected the 4600-7900 A interval.

The data were reduced using the Image Reduction and Analysis Facility (Tody 1986) in a standard way: 1) bias subtraction; 2) tracing the stellar orders; 3) scattered light subtraction; 4) order extraction for the stellar and calibration lamp frames, and 5) flat-fielding of the stellar spectra. At the end of this procedure, we measured the continuum normalised spectrum $\mathrm{S} / \mathrm{N}$ to be between 200 and 400 .

Equivalent widths were measured using the MultiProfile code (Smirnov \& Ryabchikova 1995) which approximates an observed spectrum with the sum of Gaussian profiles. The estimated error of the equivalent width measurements is in the range of $0.5-2.0 \mathrm{~m} \AA$ depending on the $\mathrm{S} / \mathrm{N}$ ratio along the spectral order.

To test the proposed atmosphere models for HD 204411 and to search for possible spectrum variability we de-archived the ELODIE spectrum (HJD $=2451685.577, R=42000)$ of this star.

The radial velocity of HD 204411 is $-14.20 \pm 0.22 \mathrm{~km} \mathrm{~s}^{-1}$ in the heliocentric reference frame based on the measurements of $76 \mathrm{Fe}$ I lines in the $S A R G$ spectrum and $-14.63 \pm 0.32 \mathrm{~km} \mathrm{~s}^{-1}$ based on $13 \mathrm{Fe}$ I lines in the ELODIE spectrum. A Gaussian fit to the observed line profiles was used to measure the position of a spectral line. These values differ from those in the literature, -10.8 to $-13.4 \mathrm{~km} \mathrm{~s}^{-1}$ (see SIMBAD database). More observations are needed to check if HD 204411 may be either a long-period binary or a spectrum variable.

\section{The global atmospheric parameters}

\subsection{Effective temperature and surface gravity}

Applying the Moon \& Dworetsky (1985) calibration to the observed Strömgren photometry extracted from the catalogue of Hauck \& Mermillod (1998) we obtained $T_{\text {eff }}=8460 \mathrm{~K}$ and $\log g=3.67$. Effective temperature is consistent with the Caliskan \& Adelman (1995) determination based on the optical spectrophotometry, while these authors obtained a lower value for the surface gravity, $\log g=3.3$. Recently, a more realistic atmospheric structure of HD 204411 was calculated (Kupka et al. 2004) with the opacity distribution functions based on the individual abundances from Caliskan \& Adelman (1995) using a modification of the Kurucz (1993) ATLAS9 code (see Piskunov \& Kupka 2001). In the absence of substantial reddening $(E(b-y)=0.01$ or $E(B-V)=0.014$, Perry et al. (1982)) the best fit to the observed optical energy distribution by Breger (1976) and by Adelman (1981) is achieved with $T_{\text {eff }}=8400 \mathrm{~K}$ and $\log g=3.5(8400 \mathrm{~g} 45)$. However, using the Lucke (1978) maps of the interstellar absorption and the distance to HD 204411 found from the Hipparcos parallax ( $\pi=$ $8.37 \pm 0.53$ mas, ESA 1997), we found that the star falls in the region with $E(B-V)$ between $0.2 \mathrm{mag} \mathrm{kpc}^{-1}$ and $0.4 \mathrm{mag} \mathrm{kpc}^{-1}$, which leads to the maximal reddening $E(B-V)=0.048$. Then, applying the dereddening procedure according to Fitzpatrick (1999) the best fit to the dereddened observed energy distribution is achieved with $T_{\text {eff }}=8700 \mathrm{~K}$ and $\log g=3.4(8700 \mathrm{~g} 34)$. A comparison between the observed spectrophotometry and the model predictions is shown in Fig. 1.

Both model atmospheres were checked using the Balmer lines. We found that the calculated $\mathrm{H} \alpha$ line profiles differ insignificantly for the two models, whereas the difference appears in $\mathrm{H} \beta$ and increases in $\mathrm{H} \gamma$. Due to its higher temperature and lower gravity the second model gives less deep profiles in the middle part of the line wings. A comparison between the observed and calculated $\mathrm{H} \gamma$ and $\mathrm{H} \beta$ line profiles for both models is presented in Fig. 2. The observed profiles of the hydrogen lines are extracted from the ELODIE spectrum. Both the $\mathrm{H} \gamma$ and $\mathrm{H} \beta$ line profiles are better fitted with the $8400 \mathrm{~g} 35$ model, which may be taken as evidence in favour of this combination of model parameters because the hydrogen lines are not 


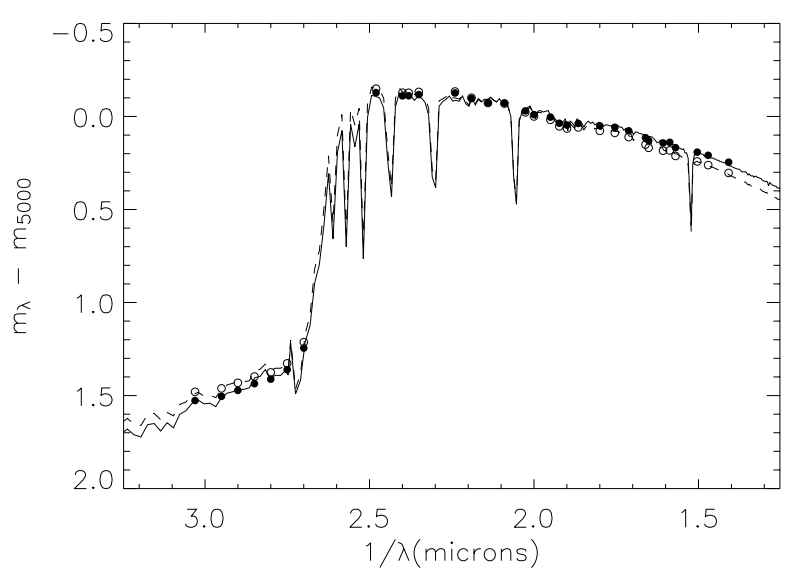

Fig. 1. Observed (Adelman 1981) and computed flux distributions. The filled circles represent the energy distribution dereddened with $E(B-V)=0.014$, the open circles represent the energy distribution dereddened with $E(B-V)=0.048$. The respective best fit theoretical calculations were obtained for the $8400 \mathrm{~g} 35$ (full line) and $8700 \mathrm{~g} 34$ (dashed line) model atmospheres.

influenced by reddening. Nevertheless, considering the problems in the continuum normalization of the hydrogen lines (e.g., in the blue wing of $\mathrm{H} \gamma$ ) we cannot totally exclude the $8700 \mathrm{~g} 34$ model. Therefore, we perform analysis of the average abundances and chemical stratification for both models. This provides a useful estimate of the errors due to the uncertainty of temperature and gravity.

We used the Hipparcos parallax to obtain the absolute magnitude and, hence, to check the evolutionary gravity. The SIMBAD database gives the $V$ magnitudes in the range of $5.28-5.32 \mathrm{mag}$ with the mean value $5.30 \pm 0.02 \mathrm{mag}$. The possible range of $E(B-V)$ is $0.014-0.048 \mathrm{mag}$. For both model atmospheres bolometric corrections were calculated from the synthetic energy distributions and $U B V$ colours using the procedure described by Buser \& Kurucz (1992). We found $B C=$ $-0.07 \mathrm{mag}$ for the $8400 \mathrm{~g} 35$ model and $B C=-0.11 \mathrm{mag}$ for the $8700 \mathrm{~g} 34$ model. These values allow us to estimate the bolometric magnitude $M_{\mathrm{bol}}=-0.28 \pm 0.24$ mag taking into account parallax, $V$ magnitude, extinction and temperature uncertainties. Then, the luminosity of HD 204411 is $\log \left(L / L_{\odot}\right)=2.01 \pm$ 0.10. Applying the modified version of the Paczynski evolutionary code with convective overshooting (see, for example, Pamyatnykh et al. 1998; Breger \& Pamyatnykh 1998) we find mass $M=2.67 \pm 0.15 M_{\odot}$ and surface gravity $\log g=3.5 \pm 0.1$. The latter agrees well with the value obtained from the spectrophotometry. The stellar radius is $R=4.6 \pm 0.2 R_{\odot}$. The position of the star in the HR diagram is shown in Fig. 3. Thus, we confirmed the advanced evolutionary status of HD 204411, which has finished its Main Sequence life.

\subsection{Magnetic field and rotational velocity}

To avoid any problems with the possible influence of magnetic field on the line profile width we estimated the rotational velocity by matching the line profile of the magnetically insensitive lines Fe I $\lambda \lambda$ 5434.52, 5576.09 (effective Landé factor $\left.g_{\text {eff }}=-0.01\right)$. Line profiles were computed with the CossAM code (Stift 2000) assuming a homogeneous atmospheric iron distribution and null microturbulent velocity. Microturbulence was deduced from the $\mathrm{Ti}, \mathrm{Cr}$ and Fe lines in the usual way: by requiring the absence of a correlation of abundance with equivalent width. All but Ti lines show a negative slope (i.e., an anti-correlation) even with the zero microturbulence.

We find that the best fit to the observed profile gives $v_{\mathrm{e}} \sin i=6.3 \pm 0.1 \mathrm{~km} \mathrm{~s}^{-1}$. The error is estimated from the $\chi_{\min }^{2}+1$ increment. However the pure rotational broadening is not representative of the observed profile (Fig.4) as quantified by a rather high $\chi^{2}=2.78$ value. In addition to the rotation and in the absence of a strong magnetic field the line broadening may be caused by macroscopic turbulence. If we adopt $v_{\mathrm{e}} \sin i=0 \mathrm{~km} \mathrm{~s}^{-1}$, the calculations show that the radial-tangential macroturbulence of $7.0 \mathrm{~km} \mathrm{~s}^{-1}$ is necessary to fit the Fe I $\lambda \lambda$ 5434.52, 5576.09 lines. While zerorotation with the macroturbulence matches the far line wings better than the rotationally broadened line profile, the line core is better reproduced by rotation alone. We then tried a combination of the rotational and macroturbulent broadening. The best fit to the Fe I $\lambda$ 5434.52, 5576.09 line profiles gives $v_{\mathrm{e}} \sin i=5.4 \mathrm{~km} \mathrm{~s}^{-1}$ and the radial-tangential macroturbulence equal to $4.8 \mathrm{~km} \mathrm{~s}^{-1}$ with $\chi^{2}=0.74$. The derived iron abundance is $\log \left(\mathrm{Fe} / N_{\text {tot }}\right)=-4.03$.

A quick inspection of the observed spectrum does not reveal any magnetic line splitting. Therefore, in the hypothesis of a non-stratified iron abundance, we have performed synthesis of the highly magnetically sensitive Fe II $\lambda 6149.248$ line ( $g_{\text {eff }}=1.35$, Mathys 1990) to estimate the surface field of HD 204411. Adopting a null value for the microturbulence and the previously determined values of the rotational and macroturbulent velocities, the best fit of the Fe I $\lambda$ 6149.258 line profile without a magnetic field gives $\chi^{2}=0.91$ for the iron abundance of $\log \left(\mathrm{Fe} / N_{\text {tot }}\right)=-4.03$ (dotted profile of Fig. 4). Adding the effect on the line profile given by a magnetic field, the best fit is obtained for an average surface field of 750 gauss tilted by $30^{\circ}$ with respect to the line of sight and an iron abundance of $\log \left(\mathrm{Fe} / N_{\text {tot }}\right)=-4.06$ (Fig. 4). Calculations were made with a 0.01 dex step in abundance, 50 gauss in the field strength, and $10^{\circ}$ in the inclination of the field with respect to the line of sight. The derived upper limit for the surface field agrees with the first measurement of the effective magnetic field $-30 \pm 11 \mathrm{G}$ recently published by Johnson (2004). From the Stokes $V$ line profiles she concluded that the field is at the crossover phase and the surface field should be relatively small.

The non-zero rotational velocity contradicts the observed possible long-period photometric variations (Adelman 2003), if the latter are connected with the rotation in the framework of the oblique rotator model of Ap stars. In principle, a rather high macroturbulence may be expected in the atmospheres of evolved stars, such as HD 204411. In this scenario it replaces rotation as the main line broadening mechanism and then the observed line broadening agrees with the long-period rotation. But if the rotation is not negligible, then the long-term photometric variations may be caused by reasons other than rotation. In the oblique rotator model, the light variations are due to a non-homogeneous distribution of abundances, which is believed to be produced by a magnetic field, on the surface 

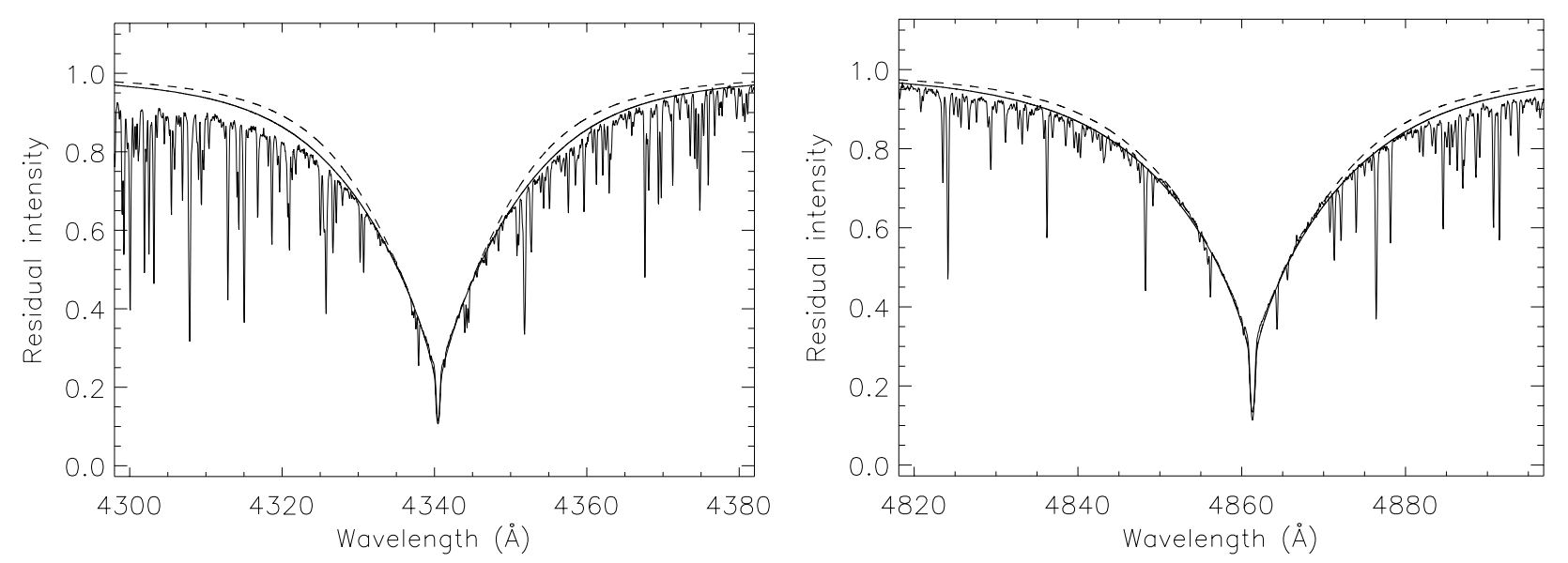

Fig. 2. A comparison between the observed (thin line) and computed (full line - the $8400 \mathrm{~g} 35$ model atmosphere, dashed line - the $8700 \mathrm{~g} 34$ model atmosphere) $\mathrm{H} \gamma$ (left panel) and $\mathrm{H} \beta$ (right panel) line profiles.

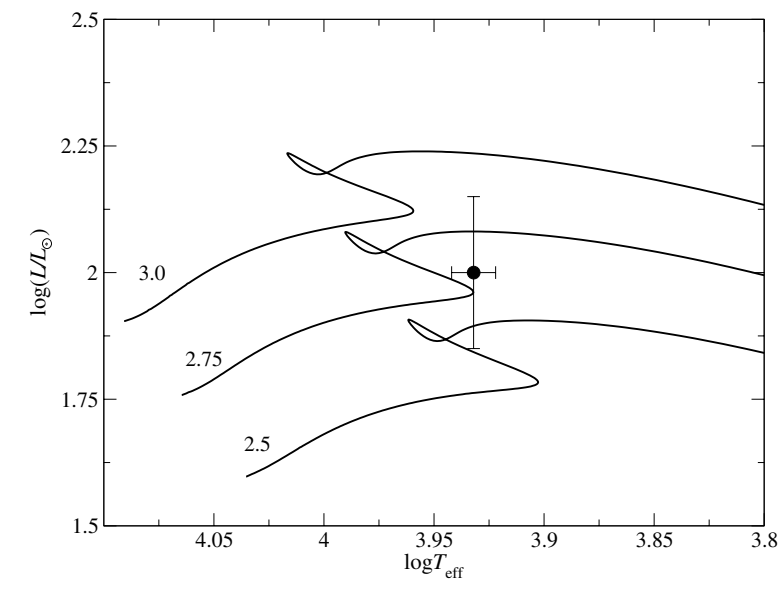

Fig. 3. Position of HD 204411 in the HR diagram.

of a rotating star. If the magnetic field is absent, the distribution of the most abundant chemical elements is expected to be homogeneous and the star should not show spectral or light variations. The only known exception from this rule is the apparently non-magnetic HgMn star $\alpha$ And, which has an inhomogeneous surface distribution of $\mathrm{Hg}$ (Adelman et al. 2002). Further photometric observations are needed to confirm the variability of HD 204411. CA already mentioned the absence of any spectral variations in their observations. To verify their conclusions we have compared the SARG and the ELODIE spectra after bringing the spectral resolution of the former to that of the latter. Figure 5 shows that the two spectra are identical and gives a strong evidence for the absence of significant spectral variations.

\section{Abundance analysis}

All atomic parameters required for the abundance calculations were extracted from VALD (Kupka et al. 1999), and some of them were corrected if necessary using the Solar Flux Atlas (Kurucz et al. 1984) (see Bikmaev et al. 2002 for the details of this procedure). A detailed description of the VALD content is given by Ryabchikova et al. (1999). Several other
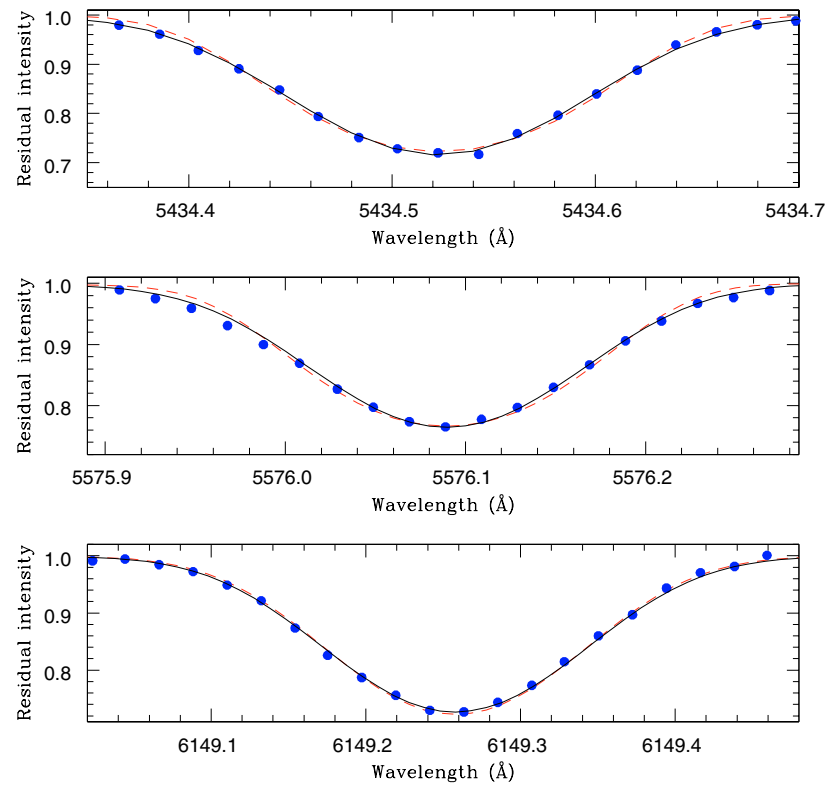

Fig. 4. The observed (circles) and computed line profiles. For the magnetically insensitive FeI $\lambda 5434.52$ line (top panel) and Fe I $\lambda 5576.09$ line (middle panel), the rotationally broadened line profiles $\left(v_{\mathrm{e}} \sin i=6.3 \mathrm{~km} \mathrm{~s}^{-1}-\right.$ dashed line) and the profiles (solid line) computed assuming $v_{\mathrm{e}} \sin i=5.4 \mathrm{~km} \mathrm{~s}^{-1}$ and $v_{\text {macro }}=4.8 \mathrm{~km} \mathrm{~s}^{-1}$ are shown. The bottom panel shows that the observed profile of Fe I $\lambda 6149.258$ line $\left(g_{\text {eff }}=1.35\right)$ is still slightly more broadened than the profile computed with the previous values of the rotational and macroturbulent velocities (dotted line). It is possible to improve the fit by adding a 750 gauss magnetic field (solid line).

sources of the transition probabilities used in our study will be discussed below for individual elements. Due to the absence of a strong magnetic field able to induce a magnetic intensification of spectral lines (Babcock 1949; Stift \& Leone 2003), and to low rotational velocity which reduces line blending, we used equivalent widths for the abundance calculations. Abundances were derived using the Kurucz WIDTH9 program modified by V. Tsymbal (private communication) to accept the VALD output format. Only unblended spectral lines were chosen for the equivalent width analysis with the help of preliminary 


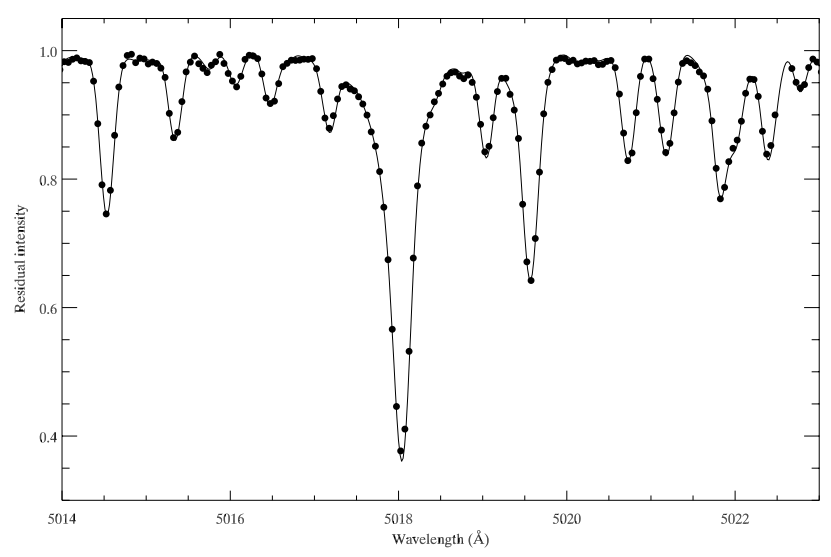

Fig. 5. A comparison between the ELODIE spectrum (circles) and the $S A R G$ spectrum (line) convolved with the $F W H M=0.11 \AA$ Gaussian profile.

spectrum synthesis of the entire observed region with the elemental overabundances typical for cool Ap stars. When we had to estimate the abundance of an element whose lines are blended, we performed a spectrum synthesis with the SYNTH3 code written by O. Kochukhov. This code is a modification of the SYNTH code by Piskunov (1992) optimized for fast calculation of the theoretical stellar spectra.

The mean abundances derived for the atmosphere of HD 204411 are given in Table 1 for the two model atmospheres discussed in Sect. 3. This table compares the abundances with those from the CA analysis. The last column contains the solar atmospheric abundances (Grevesse \& Sauval 1998), complemented by the recent data on $\mathrm{C}, \mathrm{N}, \mathrm{O}, \mathrm{Mg}, \mathrm{Si}$ (Holweger 2001), and Ba (Mashonkina \& Gehren 2000). The increase of the effective temperature by $300 \mathrm{~K}$ and the decrease of the gravity by 0.1 dex changes mainly abundances from the neutral species up to +0.3 dex as well as abundances for the elements beyond the Fe-peak. $\mathrm{Ca}, \mathrm{Mn}$ and $\mathrm{Fe}$ are closer to ionization equilibrium with the second (hotter) model, whereas other elements, such as $\mathrm{Mg}$, $\mathrm{Si}$ and $\mathrm{Ti}$, are better matched with the cooler model. The standard deviation does not change significantly from one model to another. This scatter in the abundances derived from different lines remains rather high compared to the results of the abundance analysis of the normal A-star HD 32115 which was carried out with similar approach and similar atomic data and where the typical standard deviation did not exceed $0.10-0.15$ dex for most elements (see Bikmaev et al. 2002). Even in the case of the formal ionization balance achieved for Fe with the $8700 \mathrm{~g} 34$ model, there remains a systematic discrepancy of up to 1.0 dex between the strong low- and weak high-excitation Fe II lines, which provides evidence for possible abundance stratification in the stellar atmosphere.

\subsection{Light elements: $C$ to $\mathrm{Ca}$}

Using the OI7773 triplet Gerbaldi et al. (1989) derived the oxygen abundance $\log \left(\mathrm{O} / N_{\text {tot }}\right)=-4.83 \pm 0.04$ with $T_{\text {eff }}=9000 \mathrm{~K}$. This is significantly lower than our abundance obtained from the red lines $\lambda \lambda 6156,6464$ for which negative NLTE corrections are smaller than 0.1 dex in absolute value (Takeda 1997; Przybilla et al. 2000). The difference in the adopted effective temperatures could not be a reason for the discrepancy because the triplet lines are not very sensitive to the temperature in the $8500-9000 \mathrm{~K}$ region. SARG spectra suffer from substantial fringing in the $7775 \AA$ spectral region, therefore we did not use IR-triplet in our abundance analysis. $\mathrm{C}$ and $\mathrm{O}$ are underabundant which is a typical characteristic of the CrEu stars (Roby \& Lambert 1990). The nitrogen abundance is rather uncertain. For $\mathrm{Mg}$, $\mathrm{Si}$ and $\mathrm{Ca}$ we performed stratification analysis (Sect. 5).

\subsection{Iron peak elements: Sc to Ni}

The spectrum of HD 204411 is extremely rich in the lines of neutral and ionized $\mathrm{Ti}, \mathrm{Cr}$ and $\mathrm{Fe}$. We could measure many $\mathrm{Cr}$ II and $\mathrm{Fe}$ II lines originating from the high-excitation levels with $E_{i} \geq 10 \mathrm{eV}$. There are no experimental oscillator strengths for such transitions. The two sets of calculations are available: the Kurucz (1993) GFIRON list, which is included in the current release of the VALD database, and the transition probabilities calculated with the orthogonal operator technique (Raassen \& Uylings 1998 - RU) ${ }^{1}$. We checked both sets of the theoretical oscillator strengths with our data. The RU data give slightly better results for Fe II and are much better for $\mathrm{Cr}$ II. For a set of $46 \mathrm{Cr}$ II lines the standard deviation decreases from 0.29 dex to 0.21 dex if the RU data are used. The better agreement of the RU calculations with the experimental data for Fe II was mentioned by Pickering et al. (2001). RU oscillator strengths give an $\sim 0.15$ dex higher average $\mathrm{Cr}$ abundance, but in our study the internal accuracy of the data set is more important. Therefore, we used the RU oscillator strengths for the usual abundance and for the stratification analyses. A set of neutral and ionized lines with different intensities and excitation potentials was used for stratification analysis (see Sect. 5).

Sc is deficient in HD 204411, whereas Ti, Mn, Fe and especially $\mathrm{Cr}$ are overabundant. We checked the influence of the hyperfine splitting (hfs) on the inferred Mn abundance by means of the Mn I $\lambda \lambda 4754,4783,6013,6021$ lines. The hfs constants were taken from Biehl (1976). A decrease of the Mn abundance due to hfs is from 0.25 dex for the strongest 4754,4783 $\left(W_{\lambda} \approx 50 \mathrm{~m} \AA\right.$ ) lines to less than $0.1 \mathrm{dex}$ for the 6013, 6021 lines with $W_{\lambda} \approx 25 \mathrm{~m} \AA$. Unfortunately no hfs data are available for Mn II lines used in our study. If we base Mn abundance on 8 lines with $W_{\lambda} \leq 10 \mathrm{~m} \AA$ then $\log \left(\mathrm{Mn} / N_{\text {tot }}\right)=-5.89 \pm 0.15$ (8400g35 model), which is close to that derived from the Mn I lines, therefore derived ionization imbalance and a large scatter for Mn II is caused mainly by not taking into account the hyperfine splitting. No vanadium lines are measured in our spectrum. To estimate the vanadium abundance we have used equivalent widths published by Cowley et al. (1978). Vanadium is deficient by 0.7 dex compared to the normal A star HD 32115 (Bikmaev et al. 2002) and the Sun. The $\mathrm{Cr}$ and Fe abundances conform to the effective temperature dependence found for Ap stars by Ryabchikova et al. (2004).

\footnotetext{
${ }^{1}$ ftp://ftp.wins.uva.nl/pub/orth
} 
Table 1. Atmospheric abundances in the Cr-type star HD 204411 with the error estimates based on $n$ measured lines.

\begin{tabular}{|c|c|c|c|c|c|}
\hline \multirow[t]{2}{*}{ Ion } & \multicolumn{2}{|l|}{$8400 \mathrm{~g} 35$} & \multirow{2}{*}{$\begin{array}{l}8700 \mathrm{~g} 34 \\
\log \left(N / N_{\text {tot }}\right)\end{array}$} & \multirow{2}{*}{$\begin{array}{c}\text { CA } \\
\log \left(N / N_{\text {tot }}\right)\end{array}$} & \multirow{2}{*}{$\begin{array}{c}\odot \\
\log \left(N / N_{\text {tot }}\right)\end{array}$} \\
\hline & $\log \left(N / N_{\text {tot }}\right)$ & $n$ & & & \\
\hline $\mathrm{CI}$ & $-4.37 \pm 0.17$ & 5 & $-4.19 \pm 0.17$ & & -3.45 \\
\hline N I & $-3.93 \pm 0.37$ & 3 & $-3.94 \pm 0.37$ & & -4.11 \\
\hline O I & $-4.03 \pm 0.33$ & 4 & $-4.05 \pm 0.33$ & & -3.30 \\
\hline $\mathrm{Na} I$ & $-5.28 \pm 0.20$ & 4 & $-5.01 \pm 0.24$ & & -5.71 \\
\hline $\operatorname{Mg} \mathbf{I}$ & $-4.34 \pm 0.22$ & 6 & $-4.07 \pm 0.21$ & -4.8 & -4.50 \\
\hline Mg II & $-4.62 \pm 0.55$ & 3 & $-4.67 \pm 0.63$ & -4.8 & -4.50 \\
\hline Al II & -5.85 & 1 & -5.85 & & -5.57 \\
\hline Si I & $-4.13 \pm 0.11$ & 7 & $-3.90 \pm 0.11$ & -4.4 & -4.50 \\
\hline Si II & $-4.11 \pm 0.07$ & 2 & $-4.23 \pm 0.07$ & -4.5 & -4.50 \\
\hline S I & $-5.17 \pm 0.46$ & 3 & $-4.94 \pm 0.46$ & & -4.71 \\
\hline $\mathrm{Ca} \mathrm{I}$ & $-5.17 \pm 0.02$ & 5 & $-4.80 \pm 0.04$ & -5.2 & -5.68 \\
\hline Ca II & $-4.67 \pm 0.17$ & 5 & $-4.55 \pm 0.17$ & -5.2 & -5.68 \\
\hline Sc II & -9.52 & 1 & -9.36 & -9.6 & -8.87 \\
\hline Ti I & $-6.46 \pm 0.09$ & 13 & $-6.15 \pm 0.09$ & -6.5 & -7.02 \\
\hline Ti II & $-6.49 \pm 0.16$ & 16 & $-6.36 \pm 0.16$ & -6.5 & -7.02 \\
\hline \multicolumn{6}{|l|}{$\mathrm{V}_{\mathrm{I}}$} \\
\hline $\mathrm{CrI}$ & $-4.85 \pm 0.16$ & 52 & $-4.58 \pm 0.15$ & -5.0 & -6.37 \\
\hline Cr II & $-4.70 \pm 0.21$ & 48 & $-4.65 \pm 0.21$ & -5.0 & -6.37 \\
\hline Mn I & $-5.96 \pm 0.11$ & 9 & $-5.68 \pm 0.12$ & -6.0 & -6.65 \\
\hline Mn II & $-5.66 \pm 0.38$ & 14 & $-5.63 \pm 0.40$ & -6.0 & -6.65 \\
\hline Fe I & $-3.76 \pm 0.22$ & 100 & $-3.48 \pm 0.21$ & -4.0 & -4.54 \\
\hline Fe II & $-3.52 \pm 0.33$ & 83 & $-3.53 \pm 0.26$ & -4.0 & -4.54 \\
\hline Co I & $-6.19 \pm 0.28$ & 3 & $-5.90 \pm 0.30$ & -6.5 & -7.12 \\
\hline Co II & -6.50 & 1 & -6.50 & -6.5 & -7.12 \\
\hline Ni I & $-5.68 \pm 0.22$ & 28 & $-5.41 \pm 0.22$ & -5.7 & -5.79 \\
\hline Ni II & $-5.31 \pm 0.12$ & 2 & $-5.31 \pm 0.14$ & -5.7 & -5.79 \\
\hline $\mathrm{Zn} \mathrm{I}$ & $-7.74 \pm 0.04$ & 2 & $-7.46 \pm 0.04$ & & -7.44 \\
\hline Sr II & -8.5 & 2 & -8.0 & -8.5 & -9.07 \\
\hline Y II & $-9.95 \pm 0.28$ & 5 & $-9.74 \pm 0.29$ & -9.9 & -9.80 \\
\hline Zr II & -8.66 & 1 & -8.52 & -9.0 & -9.44 \\
\hline Ba II & $-9.02 \pm 0.28$ & 3 & $-8.60 \pm 0.31$ & -8.9 & -9.83 \\
\hline $\mathrm{Ce}$ II & -10.26 & 1 & -9.97 & & -10.46 \\
\hline Pr II & $<-10.5$ & 1 & $<-10.5$ & -9.5 & -11.33 \\
\hline Pr III & $<-10.5$ & 1 & $<-10.2$ & & -11.33 \\
\hline Nd II & $-9.48 \pm 0.27$ & 3 & $-9.12 \pm 0.28$ & -9.5 & -10.54 \\
\hline Nd III & $-10.05 \pm 0.10$ & 2 & $-9.95 \pm 0.14$ & & -10.54 \\
\hline Eu II & -10.95 & 1 & -10.67 & -11.0 & -11.53 \\
\hline $\bar{T} \overline{\text { eff }}$ & 8400 & & 8700 & 8500 & 5777 \\
\hline $\log g$ & 3.50 & & 3.40 & 3.30 & 4.44 \\
\hline
\end{tabular}

\section{3. s-process elements: $S r, Y, Z r, B a$}

There are no measurable $\mathrm{Sr}$ lines in the investigated spectral region of the $S A R G$ spectrum. The strontium abundance was obtained by fitting the $\operatorname{Sr} I I \lambda$ 4215, 4305 lines in the ELODIE spectrum. Both lines are well matched with the strontium abundance exceeding the solar one by 0.5 dex, which coincides with the CA result. For the hotter model atmosphere we need a 1.0 dex overabundance for $\mathrm{Sr}$ to fit the observed line profiles. The yttrium abundance agrees well with the CA results and is solar within the error bars. The zirconium abundance was estimated with one weak $\mathrm{Zr}$ II line and is rather uncertain, therefore we may only say that it does not contradict the CA results based on the stronger blue $\mathrm{Zr}$ II lines. Within the error limits the $\mathrm{Ba}$ abundance agrees with the $\mathrm{CA}$ value, and is only slightly higher than the solar one. Sr, Y and $\mathrm{Zr}$ abundances in different groups of Ap stars were studied by Allen (1977). Our results for HD 204411 agree with his conclusion that in cool Ap stars yttrium is depleted relative to the neighbour elements $\mathrm{Sr}$ and $\mathrm{Zr}$. We note that the Ba II lines all show asymmetric profiles, with the red wing being more extended than the blue one. This effect is especially prominent in the weakest Ba II $\lambda 5853$ line and cannot be explained by the isotopic or hyperfine splitting. More high resolution high $\mathrm{S} / \mathrm{N}$ observations are needed to search for the possible reason for the observed asymmetry.

\subsection{Rare-earth elements}

The weakness of the lanthanide's (rare-earth) spectrum was emphasized in all previous abundance studies of HD 204411 (Sargent et al. 1969; CA), but a disagreement in individual 

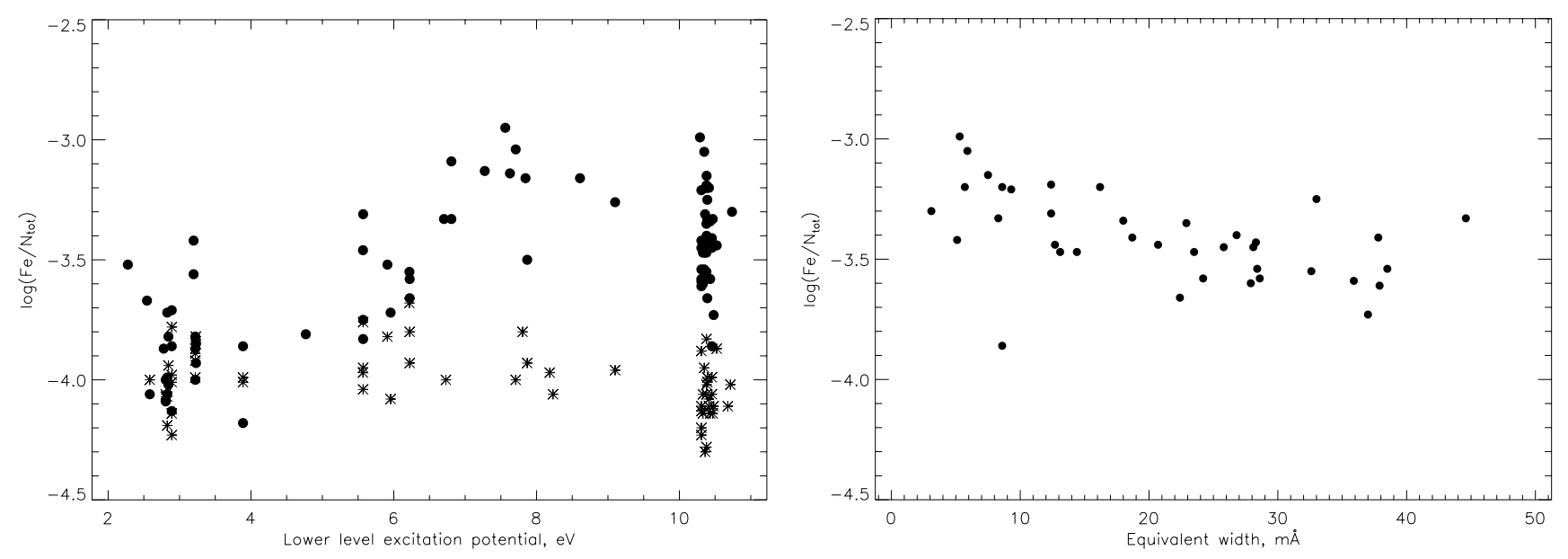

Fig. 6. Dependence of the individual abundances on the excitation potential for Fe II lines (left panel) in HD 204411 (filled circles) and Sirius (asterisks). Dependence of the individual abundances on the equivalent width for Fe II lines with $E_{i}>10 \mathrm{eV}$ in $\mathrm{HD} 204411$ (right panel).

elemental abundances still existed. The new experimental transition probabilities for Nd II lines were taken from Den Hartog et al. (2003), while the theoretical calculations for Nd III (Bord 2000) and Pr III (Bord, private communication) were used. For Ce II $\lambda 4628.16$, the oscillator strength, $\log g f=0.20$, was taken from Palmeri et al. (2000) and for other rare-earth elements transition probabilities were extracted from VALD. Eu abundance was derived using one line Eu II $\lambda$ 6645.11. Our results for Nd II and Eu II coincide with CA and support an extremely small overabundance of the REE in HD 204411 compared to other Ap stars. The two Nd III lines $\lambda \lambda 5203$ and 6327 measured in the SARG spectrum show even lower $\mathrm{Nd}$ abundance than derived from the blue Nd II lines $\lambda \lambda 4059$, 4061 and 4706. CA derived $\log \left(\operatorname{Pr} / N_{\text {tot }}\right)=-9.5$ from $\operatorname{Pr}$ II lines. The Pr abundance is usually based on the strongest $\operatorname{Pr}$ II $\lambda 4222.934$ line in the blue spectral range. This line is blended with the high-excitation Fe II $\lambda 4222.881\left(E_{i}=11 \mathrm{eV}\right)$ line, which contributes more than $50 \%$ to the blend. Taking this line into account we could match the observed feature with $\log \left(\operatorname{Pr} / N_{\text {tot }}\right)=-10.5$, which agrees with the upper limit for the strongest Pr III $\lambda 6090$ line. The inclusion of the hyperfine structure for the Pr II $\lambda 4222.934$ line (Ginibre 1989) does not change Pr abundance for HD 204411. The abundance difference for Pr between this study and that of CA may lie in the treatment of this high excitation Fe II line. We also checked rather high $\mathrm{Ce}$ and $\mathrm{Gd}$ abundances reported by Sargent et al. (1969) using their equivalent widths and the new atomic data. We found that in all cases they used blended lines where the main contribution came from the lines other than Ce II or Gd II.

\section{Abundance stratification}

Due to the high average $\mathrm{Cr}$ and $\mathrm{Fe}$ overabundances, many of the high-excitation $\mathrm{Cr}$ II and Fe II lines are observed in the spectrum of HD 204411. In particular, the Fe II lines with $E_{i}>7 \mathrm{eV}$ give higher iron abundance than spectral lines with $E_{i}<7 \mathrm{eV}$. This is shown in the left panel of Fig. 6. Furthermore, the spectral lines with $E_{i}>10 \mathrm{eV}$ do not give consistent abundances. On the right panel of Fig. 6 we show that for this group of lines the individual abundance decreases with the increase of equivalent width. Both plots provide evidence that deeper in the atmosphere the iron abundance is higher, i.e. Fe is stratified in the atmosphere of HD 204411. We checked the possibility for the observed dependences to be caused by the uncertainties of the transition probabilities. High-excitation lines are observed in Sirius spectrum. We have measured them using the Sirius Flux Atlas, kindly provided by R. Kurucz. Abundance calculations were performed with the ATLAS 12 model of Sirius ${ }^{2}$. The results for Sirius are shown in Fig. 6 with asterisks. We conclude that in the whole range of excitation potentials the accuracy of the RU oscillator strengths is about the same and is not worse than \pm 0.15 dex. Since no microturbulence is evident in the mean abundance analysis of HD 204411, any depthdependent microturbulent velocity is a very unlikely reason for the discrepant behaviour of the high-excitation lines.

Usually the vertical abundance stratification manifests itself as an impossibility to fit the line core and line wings in strong lines with developed Stark wings (Ca II K, Si II, Mg II lines, see Babel 1992) with the same abundance or as an impossibility to describe the low and high-excitation lines with a chemically homogeneous atmosphere (Ryabchikova et al. 2003). We studied abundance stratification in the atmosphere of HD 204411 using two approaches. The first one, based on trial and error calculations, is the same as we used in the stratification study of $\gamma$ Equ (Ryabchikova et al. 2002). The second approach has made use of the DDAFIT - a newly developed automatic procedure for determination of vertical abundance gradients. This program is written in IDL and provides an optimization and visualization interface to the spectrum synthesis calculations with SYNTH3. Vertical abundance distributions are described with four parameters: chemical abundance in the upper atmosphere, abundance in deep layers, the vertical position of abundance step and the width of the transition region where chemical abundance changes between the two values. All four parameters can be optimized simultaneously with the least-squares fitting routine and based on observations of unlimited number of spectral regions, possibly using different weights in accordance with the quality or relative

\footnotetext{
${ }^{2}$ http://cfaku5.cfa.harvard.edu/Stars/SIRIUS
} 
Table 2. A list of spectral lines used for the stratification calculations with the excitation potential (eV), oscillator strength (log $g f)$ and Stark damping constant $\left(\log \gamma_{\mathrm{St}}\right)$.

\begin{tabular}{|c|c|c|c|c|c|c|c|c|c|c|c|}
\hline Ion & Wavelength & $E_{i}(\mathrm{eV})$ & $\log g f$ & $\log \gamma_{\mathrm{St}}$ & Ref. & Ion & Wavelength & $E_{i}(\mathrm{eV})$ & $\log g f$ & $\log \gamma_{\mathrm{St}}$ & Ref. \\
\hline$\overline{M g} \mathrm{I}$ & 4702.991 & 4.346 & -0.666 & -4.46 & $\mathrm{LZ}$ & CrI & 5298.494 & 2.900 & -0.350 & -3.749 & K88 \\
\hline Mg I & 4730.029 & 4.346 & -2.409 & appx & JKPS & Cr II & 5305.865 & 3.827 & -2.160 & -6.599 & RU \\
\hline Mg II & 4739.593 & 11.569 & -0.660 & appx & $\mathrm{KP}$ & Cr II & 5305.929 & 10.760 & -0.170 & -5.348 & RU \\
\hline Mg II & 4739.709 & 11.569 & -0.820 & appx & $\mathrm{KP}$ & Cr II & 5308.425 & 4.071 & -2.060 & -6.639 & RU \\
\hline $\mathrm{Mg}_{\mathrm{I}}$ & 5172.684 & 2.712 & -0.402 & -5.47 & $\mathrm{AZ}$ & CrI & 5344.757 & 3.449 & -1.060 & -5.344 & MFW \\
\hline Mg I & 5528.405 & 4.346 & -0.620 & -4.46 & $\mathrm{LZ}$ & CrI & 5348.315 & 1.004 & -1.290 & -6.112 & MFW \\
\hline $\operatorname{Mg} I$ & 5711.088 & 4.346 & -1.833 & appx & $\mathrm{LZ}$ & Cr II & 5564.716 & 8.227 & -2.080 & -5.670 & RU \\
\hline Mg I & 6318.717 & 5.108 & -1.730 & appx & $\mathrm{KP}$ & Cr II & 5564.741 & 10.893 & 0.510 & -5.364 & RU \\
\hline Mg II & 6346.742 & 11.569 & 0.020 & -3.50 & $\mathrm{KP}$ & Cr II & 5569.110 & 10.872 & 0.860 & -5.359 & RU \\
\hline Mg II & 6346.964 & 11.569 & -0.140 & -3.50 & $\mathrm{KP}$ & Cr II & 5569.617 & 10.904 & 0.710 & -5.358 & RU \\
\hline \multirow[t]{2}{*}{ Mg II } & 7896.366 & 9.999 & 0.650 & -4.54 & $\mathrm{KP}$ & Cr II & 6050.242 & 11.098 & 0.210 & -4.683 & RU \\
\hline & & & & & & Cr II & 6053.466 & 4.745 & -2.220 & -6.633 & RU \\
\hline Si II & 5055.984 & 10.074 & 0.593 & -4.78 & SG & Cr II & 6068.023 & 6.686 & -1.736 & -6.559 & K88 \\
\hline Si II & 5056.317 & 10.074 & -0.359 & -4.78 & SG & Cr II & 6138.721 & 6.484 & -2.150 & -6.728 & RU \\
\hline Si I & 5701.104 & 4.930 & -2.000 & -4.41 & GARZn & Cr II & 6147.154 & 4.756 & -2.890 & -6.656 & RU \\
\hline Si I & 5708.400 & 4.954 & -1.320 & -4.41 & GARZn & Cr II & 6336.263 & 4.073 & -3.760 & -6.638 & RU \\
\hline Si I & 5772.146 & 5.082 & -1.600 & -4.06 & GARZn & & & & & & \\
\hline Si II & 5957.559 & 10.067 & -0.301 & -4.84 & SG & Fe II & 5018.440 & 2.891 & -1.340 & -6.585 & RU \\
\hline Si II & 5978.930 & 10.074 & 0.004 & -4.84 & SG & $\mathrm{Fe}$ II & 5018.669 & 6.138 & -4.010 & -6.537 & RU \\
\hline Si I & 6142.483 & 5.619 & -1.420 & appx & astr & $\mathrm{Fe} \mathrm{I}$ & 5019.160 & 4.580 & -2.080 & -5.895 & GK \\
\hline \multirow[t]{2}{*}{ Si II } & 6347.109 & 8.121 & 0.297 & -5.04 & $\mathrm{BBCB}$ & Fe II & 5019.462 & 5.569 & -2.780 & -6.607 & RU \\
\hline & & & & & & $\mathrm{Fe} I$ & 5269.537 & 0.859 & -1.321 & -6.300 & BPS1 \\
\hline Ca II & 4716.743 & 7.047 & -2.490 & -4.449 & astr & $\mathrm{Fe}$ II & 5278.938 & 5.911 & -2.680 & -6.696 & RU \\
\hline Ca II & 4721.022 & 7.050 & -2.330 & -4.449 & astr & $\mathrm{Fe} I$ & 5281.790 & 3.038 & -0.834 & -5.489 & BWLW \\
\hline Ca II & 4799.973 & 8.438 & -0.420 & -2.915 & TB & $\mathrm{Fe}$ II & 5291.666 & 10.480 & 0.540 & -5.468 & RU \\
\hline Ca II & 5021.138 & 7.515 & -1.217 & -4.612 & BWL & Fe II & 5303.395 & 8.185 & -1.530 & -5.822 & RU \\
\hline Ca II & 5339.188 & 8.438 & -0.079 & -3.700 & $\mathrm{~TB}$ & Fe II & 5325.553 & 3.221 & -3.320 & -6.603 & RU \\
\hline Ca I & 6439.075 & 2.526 & 0.390 & -6.072 & SR & $\mathrm{Fe} \mathrm{I}$ & 5326.142 & 3.573 & -2.071 & -6.209 & BK \\
\hline $\mathrm{CaI}$ & 6449.808 & 2.521 & -0.502 & -6.071 & SR & $\mathrm{Fe} I$ & 5434.523 & 1.011 & -2.122 & -6.303 & BPS1 \\
\hline $\mathrm{Ca} \mathrm{I}$ & 6455.598 & 2.523 & -1.290 & -6.072 & SR & $\mathrm{Fe} \mathrm{I}$ & 5560.211 & 4.434 & -1.090 & -4.323 & MRW \\
\hline Ca II & 6456.875 & 8.438 & 0.410 & -3.700 & TB & Fe II & 5567.842 & 6.730 & -1.870 & -6.578 & RU \\
\hline \multirow[t]{2}{*}{$\mathrm{Ca} \mathrm{I}$} & 6462.567 & 2.523 & 0.262 & -6.072 & SR & $\mathrm{Fe} I$ & 5576.088 & 3.430 & -0.900 & -5.491 & MRW \\
\hline & & & & & & $\mathrm{Fe}$ II & 5961.705 & 10.678 & 0.670 & -4.950 & RU \\
\hline Cr II & 5046.429 & 8.227 & -1.740 & -5.909 & RU & Fe II & 6071.426 & 10.714 & -0.250 & -5.640 & RU \\
\hline CrI & 5265.148 & 3.428 & -0.529 & -5.324 & K88 & $\mathrm{Fe} I$ & 6136.615 & 2.453 & -1.400 & -6.327 & BPSS \\
\hline Cr I & 5296.691 & 0.983 & -1.400 & -6.120 & MFW & $\mathrm{Fe} I$ & 6136.994 & 2.198 & -2.950 & -6.196 & BPSS \\
\hline Cr I & 5297.377 & 2.900 & 0.167 & -4.307 & MFW & $\mathrm{Fe} I$ & 6137.691 & 2.588 & -1.403 & -6.112 & BPS2 \\
\hline Cr II & 5297.606 & 10.754 & -0.320 & appx & RU & Fe II & 6150.098 & 3.221 & -4.820 & -6.678 & RU \\
\hline CrI & 5298.016 & 2.900 & -0.060 & -4.051 & MFW & $\mathrm{Fe} \mathrm{I}$ & 6335.330 & 2.198 & -2.177 & -6.195 & BWLW \\
\hline Cr I & 5298.272 & 0.983 & -1.150 & -6.117 & MFW & $\mathrm{Fe} \mathrm{I}$ & 6336.824 & 3.686 & -0.856 & -5.467 & $\mathrm{BK}$ \\
\hline
\end{tabular}

LZ - Lincke \& Ziegenbein (1971); JKPS - Jönsson et al. (1984); KP - Kurucz \& Peytremann (1975); AZ - Andersen et al. (1967); SG Schulz-Gulde (1969); GARZn - Garz (1974 - corrected); BBCB - Berry et al. (1971); TB - TOPBASE (Seaton et al. 1992); BWL - Black et al. (1972); SR - Smith \& Raggett (1981); RU - Raassen \& Uylings (1998); K88 - Kurucz (1988); MWF - Martin et al. (1988); GK - Gurtovenko \& Kostyk (1981); MRW - May et al. (1974); BPS1 - Blackwell et al. (1979); BPSS - Blackwell et al. (1982a); BPS2 - Blackwell et al. (1982b); BWLW - O’Brian et al. (1991); BK - Bard \& Kock (1994).

importance of the observations of particular spectral features. The program derives one chemical stratification profile at a time, but is able to account for any number of fixed stratified abundances of, e.g., chemical elements blending lines of interest. The use of the step function to model the abundance distribution is justified by the results of the diffusion calculations for
$\mathrm{Ca}, \mathrm{Ti}, \mathrm{Cr}, \mathrm{Fe}, \mathrm{Sr}$ in the Ap star 53 Cam (Babel 1992) and the recent self-consistent model diffusion calculations (LeBlanc $\&$ Monin 2004). We found that the trial and error and automatic methods produce nearly the same abundance distribution. For instance, with the former method we find for Fe that $\log \left(\mathrm{Fe} / N_{\text {tot }}\right)_{\text {upper }}=-4.7, \log \left(\mathrm{Fe} / N_{\text {tot }}\right)_{\text {lower }}=-3.0$ and the jump 
Table 3. Parameters of the vertical abundance distributions.

\begin{tabular}{|c|c|c|c|c|c|c|}
\hline \multirow{2}{*}{$\begin{array}{l}\text { Model / } \\
\log (g f)_{\text {set }}\end{array}$} & \multirow[t]{2}{*}{ Element } & \multicolumn{3}{|c|}{ Abundances in $\log \left(N / N_{\text {tot }}\right)$} & \multicolumn{2}{|c|}{ Abundance jump parameters in $\log \tau_{5000}$} \\
\hline & & Initial & Upper atmosphere & Low atmosphere & Position & Width \\
\hline \multicolumn{7}{|c|}{ Pure rotation } \\
\hline $8400 \mathrm{~g} 35$ & $\mathrm{Mg}$ & -4.1 & $-3.63 \pm 0.15$ & $-5.79 \pm 2.17$ & $-0.79 \pm 1.32$ & $3.11 \pm 2.77$ \\
\hline $8700 \mathrm{~g} 34$ & $\mathrm{Mg}$ & -4.1 & $-3.13 \pm 0.11$ & $-6.07 \pm 1.21$ & $-1.75 \pm 0.70$ & $0.62 \pm 2.89$ \\
\hline $8400 \mathrm{~g} 35$ & $\mathrm{Si}$ & -4.4 & $-5.84 \pm 0.08$ & $-3.78 \pm 0.02$ & $-0.98 \pm 0.02$ & $0.07 \pm 0.10$ \\
\hline Garz+BBC & $\mathrm{Si}$ & -4.5 & $-5.49 \pm 0.08$ & $-3.61 \pm 0.02$ & $-0.82 \pm 0.18$ & $0.02 \pm 0.25$ \\
\hline $8700 \mathrm{~g} 34$ & $\mathrm{Si}$ & -4.0 & $-5.76 \pm 0.21$ & $-3.80 \pm 0.03$ & $-1.09 \pm 0.30$ & $0.09 \pm 0.45$ \\
\hline $8400 \mathrm{~g} 35$ & $\mathrm{Ca}$ & -5.3 & $-5.62 \pm 0.04$ & $-4.32 \pm 0.03$ & $-0.74 \pm 0.14$ & $0.20 \pm 0.25$ \\
\hline $8700 \mathrm{~g} 34$ & $\mathrm{Ca}$ & -5.0 & $-5.24 \pm 0.04$ & $-4.09 \pm 0.07$ & $-0.62 \pm 0.21$ & $0.13 \pm 0.64$ \\
\hline $8400 \mathrm{~g} 35$ & $\mathrm{Cr}$ & -4.8 & $-5.04 \pm 0.06$ & $-4.75 \pm 0.11$ & $-1.05 \pm 0.67$ & $0.03 \pm 3.30$ \\
\hline $8700 \mathrm{~g} 34$ & $\mathrm{Cr}$ & -4.6 & $-4.68 \pm 0.05$ & $-4.93 \pm 0.46$ & $-0.94 \pm 1.21$ & $0.38 \pm 4.63$ \\
\hline $8400 \mathrm{~g} 35$ & $\mathrm{Fe}$ & -4.0 & $-4.50 \pm 0.03$ & $-2.75 \pm 0.04$ & $-0.66 \pm 0.06$ & $0.05 \pm 0.61$ \\
\hline VALD & $\mathrm{Fe}$ & -4.0 & $-4.10 \pm 0.02$ & $-2.61 \pm 0.06$ & $-0.54 \pm 0.07$ & $0.09 \pm 4.22$ \\
\hline $8700 \mathrm{~g} 34$ & $\mathrm{Fe}$ & -3.7 & $-4.06 \pm 0.02$ & $-2.55 \pm 0.06$ & $-0.55 \pm 0.08$ & $0.04 \pm 0.38$ \\
\hline \multicolumn{7}{|c|}{ Rotation \& macroturbulence } \\
\hline $8400 \mathrm{~g} 35$ & $\mathrm{Mg}$ & -4.1 & $-2.92 \pm 0.18$ & $-4.99 \pm 0.15$ & $-2.20 \pm 0.43$ & $1.27 \pm 3.50$ \\
\hline $8700 \mathrm{~g} 34$ & $\mathrm{Mg}$ & -4.1 & $-3.42 \pm 0.15$ & $-4.83 \pm 0.10$ & $-1.84 \pm 0.58$ & $0.00 \pm 40.18$ \\
\hline $8400 \mathrm{~g} 35$ & $\mathrm{Si}$ & -4.4 & $-5.99 \pm 0.22$ & $-3.87 \pm 0.02$ & $-1.20 \pm 0.07$ & $0.08 \pm 0.34$ \\
\hline $8700 \mathrm{~g} 34$ & $\mathrm{Si}$ & -4.0 & $-5.51 \pm 0.23$ & $-3.90 \pm 0.04$ & $-1.20 \pm 1.12$ & $0.01 \pm 1.69$ \\
\hline $8400 \mathrm{~g} 35$ & $\mathrm{Ca}$ & -5.3 & $-5.49 \pm 0.04$ & $-4.33 \pm 0.04$ & $-0.70 \pm 0.16$ & $0.12 \pm 0.19$ \\
\hline $8700 \mathrm{~g} 34$ & $\mathrm{Ca}$ & -5.0 & $-5.11 \pm 0.05$ & $-4.07 \pm 0.15$ & $-0.57 \pm 0.48$ & $0.08 \pm 2.74$ \\
\hline $8400 \mathrm{~g} 35$ & $\mathrm{Cr}$ & -4.6 & $-4.83 \pm 0.43$ & $-4.90 \pm 0.04$ & $-2.40 \pm 5.22$ & $0.24 \pm 60.17$ \\
\hline $8700 \mathrm{~g} 34$ & $\mathrm{Cr}$ & -4.6 & $-4.52 \pm 0.05$ & $-4.92 \pm 0.46$ & $-1.40 \pm 0.10$ & $0.01 \pm 0.00$ \\
\hline $8400 \mathrm{~g} 35$ & $\mathrm{Fe}$ & -4.0 & $-4.47 \pm 0.03$ & $-2.94 \pm 0.06$ & $-0.72 \pm 0.09$ & $0.01 \pm 0.31$ \\
\hline $8700 \mathrm{~g} 34$ & $\mathrm{Fe}$ & -3.7 & $-3.93 \pm 0.02$ & $-2.60 \pm 0.08$ & $-0.49 \pm 0.09$ & $0.14 \pm 0.52$ \\
\hline
\end{tabular}

position at $\log \tau_{5000}=-0.75$ for the $8400 \mathrm{~g} 35$ model, which is very close to the results of the automatic fitting (Table 2). Given that the automatic reconstruction of the vertical abundance is more objective and allows us to fit a large number of spectral regions simultaneously, we present the results obtained with the automatic procedure.

A list of spectral lines used in the stratification analysis is given in Table 2. For Mg, Si, CrI and Fe I the atomic parameters were extracted from the VALD database. The oscillator strengths for Ca are taken from Smith \& Raggett (1981) for Ca I and from TOPBASE (Seaton et al. 1992) for Ca II. For Cr II and Fe II we used the oscillator strengths from Raassen \& Uylings (1998) and their database. The Stark damping constants per one electron, $\gamma_{\mathrm{St}}$, are given for $T=10000 \mathrm{~K}$. If no constant is available in VALD the approximation formula was used (Cowley 1971).

The final results of the element distribution in the atmosphere of HD 204411 are presented in Table 3 for the two atmosphere models and are illustrated in Fig. 7 for the $8400 \mathrm{~g} 35$ model. In Table 3 we give an initial uniform abundance, upper atmosphere abundance, low atmosphere abundance, position and width of the abundance jump with the respective error estimates. The position and width of the abundance jump are given in $\log \tau_{5000}$. The stratification calculations were performed for two cases of line broadening: pure rotation with $v_{\mathrm{e}} \sin i=6.3 \mathrm{~km} \mathrm{~s}^{-1}$, and a combination of the rotation and radial-tangential macroturbulence derived in Sect. 3.2. The results show that the formal stratification solution depends insignificantly on the adopted line broadening.

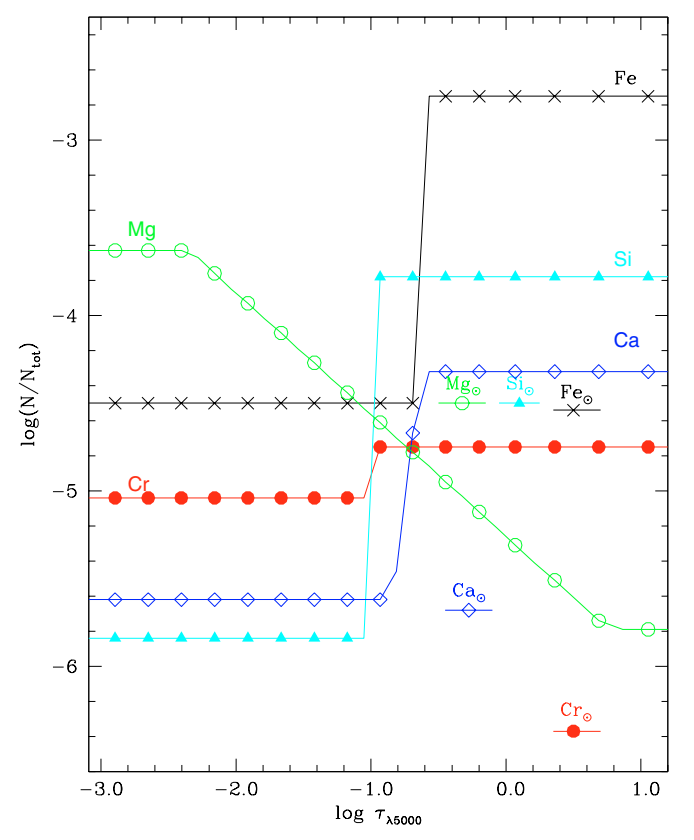

Fig. 7. Abundance stratification in the atmosphere of HD 204411.

All five elements have an abundance jumps within a small range of optical depths in the atmosphere. The width of the jump in abundance profile is also very small for four out of five elements. Below we comment on the stratification of individual elements.

Magnesium. This is the only element that shows a tendency to an abundance increase towards the outer atmospheric 

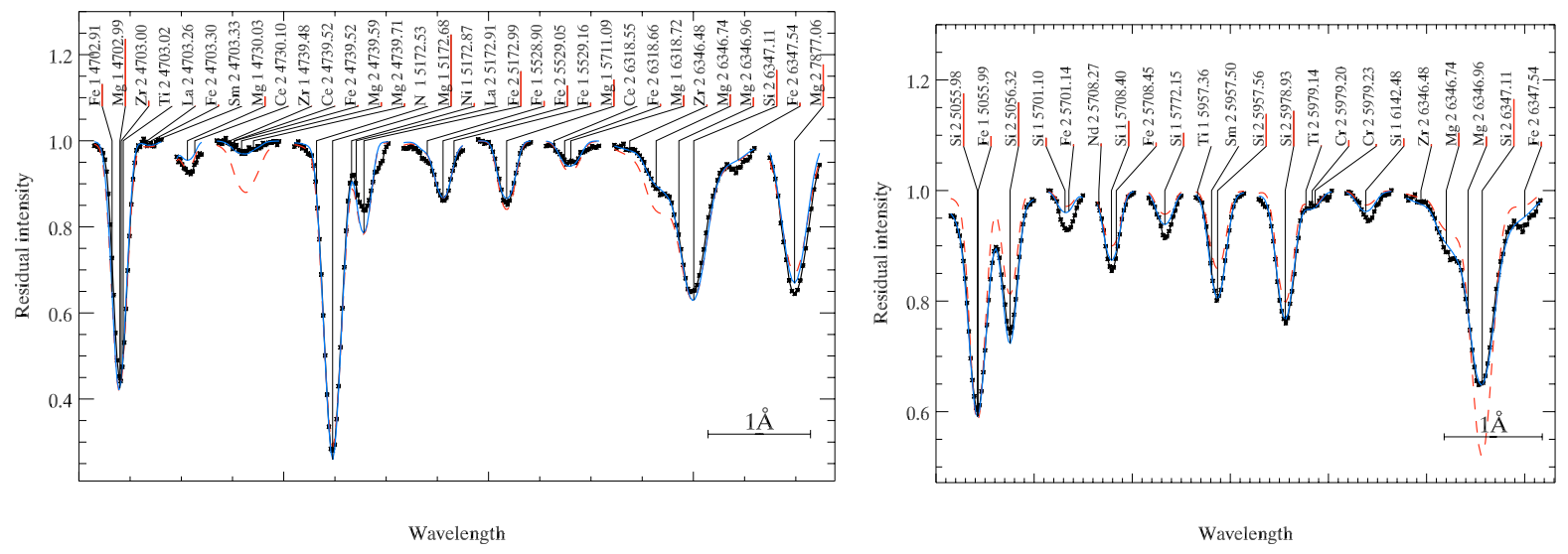

Fig. 8. A comparison between the observed line profiles and calculations with the stratified abundance distribution (full line) and with the homogeneous abundances (dashed line) from Table 3. The Mg lines (left panel) and the Si lines (right panel) are shown in this plot.

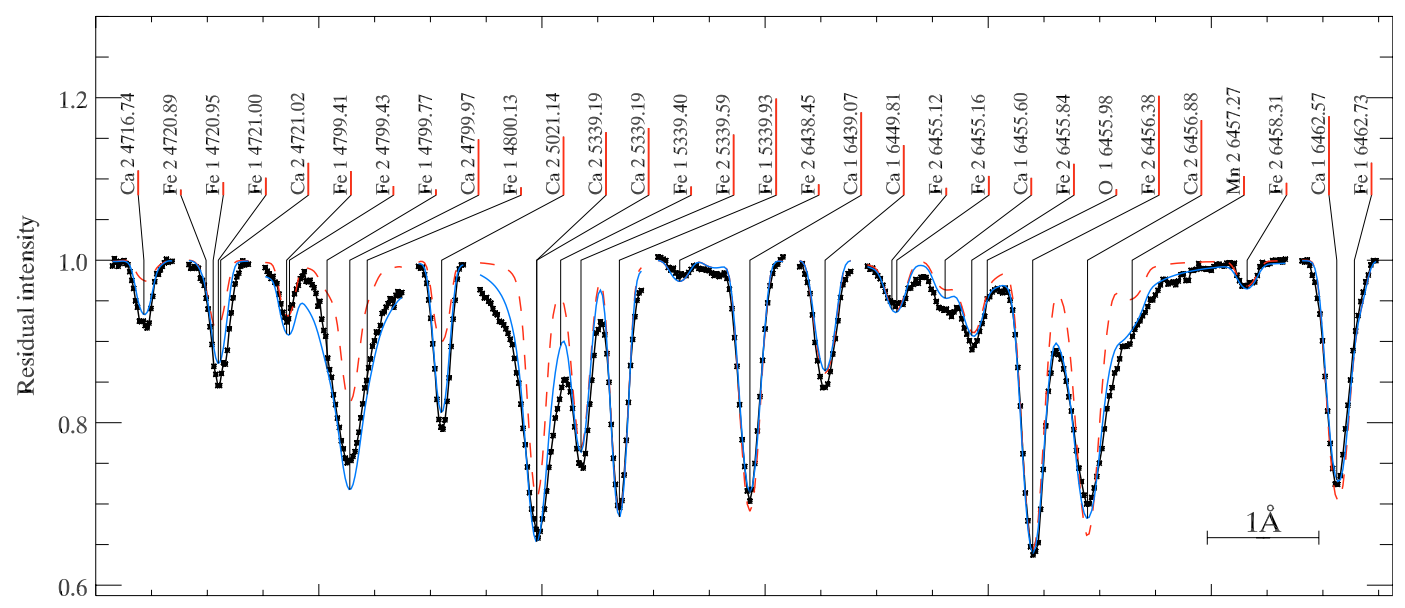

Wavelength

Fig. 9. The same as in Fig. 8 but for Ca.

layers with a rather wide transition zone. The vertical abundance inhomogeneity is mainly based on the weak highexcitation Mg II lines $\lambda \lambda$ 4739, 6346, whose transition probabilities adopted in VALD are very close to those in TOPBASE (Seaton et al. 1992). The oscillator strengths of these lines are unlikely to be the reason for the abundance disagreement in the homogeneous atmosphere. The same data were used by Przybilla et al. (2000) in an NLTE analysis of A-type stars, where they found small NLTE corrections for these lines, and derived good abundance agreement between the neutral and ionized lines in Vega, and in two A-type supergiants. If we assume a homogeneous $\mathrm{Mg}$ abundance obtained using these high-excitation lines, the NLTE corrections should be of the order of -0.5 dex for all neutral lines and -1.0 dex for the red $\mathrm{Mg}$ II $\lambda 7877$ line to reach the equilibrium magnesium abundance in HD 204411, which is rather improbable. However, the NLTE effects may change the derived Mg distribution in the atmosphere. Figure 8 (left panel) shows a fit of the computed line profiles to the observed spectrum. The dashed line represents calculations with the initial homogeneous abundance from Table 3. Stratification helps to remove a large difference in abundances derived from the strong neutral magnesium lines and the weak high-excitation $\mathrm{Mg}$ II lines including those which we did not use in the stratification analysis, e.g. Mg II $\lambda$ 5401.54. It also improves a fit of the wings of the $\mathrm{Mg}$ II 4481 doublet, observed in the ELODIE spectrum.

Silicon. For silicon, typical evidence of the abundance stratification appears in the lines of different intensities and different excitation. It is not possible to fit the cores and wings of the strong Si II lines in the HD 204411 spectrum with a unique abundance, while a good fit is obtained for the same lines in the Sirius spectrum with the abundance $\log \left(\mathrm{Si} / N_{\text {tot }}\right)=$ $-4.39 \pm 0.07$. It proves the reliability of the atomic parameters used in our study.

In HD 204411 silicon is underabundant in the upper atmosphere and overabundant in the deep layers. Figure 8 (right panel) shows a comparison between the observed and computed Si line profiles for the homogeneous and stratified atmosphere. The Si stratification is very similar in both models. The majority of the oscillator strengths for neutral lines (Garz 1973) have to be increased by +0.05 to +0.15 dex to fit the solar spectrum better. For the $\mathrm{Si}$ I $\lambda 6142.483$ line a correction of $-0.5 \mathrm{dex}$ (typical for the entire $3 \mathrm{~d}-5 \mathrm{f}$ transition array) was applied to the 


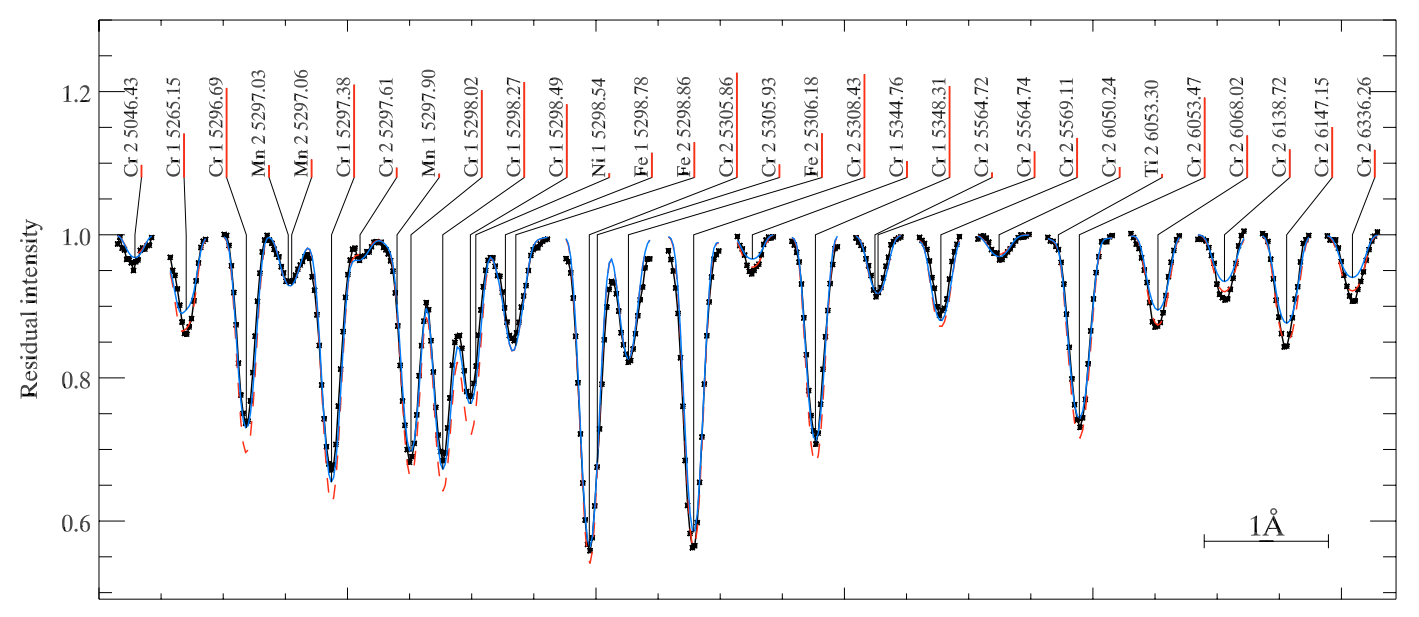

Wavelength

Fig. 10. The same as in Fig. 8 but for Cr.

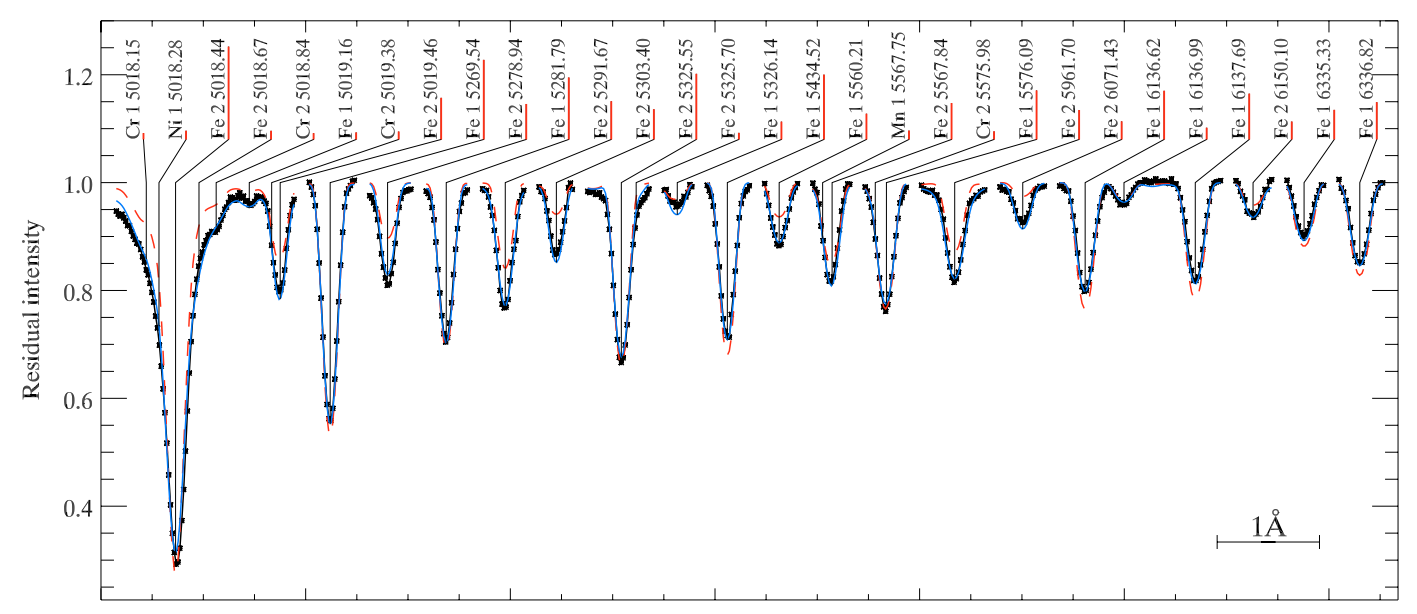

Wavelength

Fig. 11. The same as in Fig. 8 but for Fe.

KP value to fit the solar line profiles. The step model which we are using in our stratification analysis seems to be too simple for the description of the observed line profiles of the weak neutral Si lines. The standard deviation of the final fit for the $8400 \mathrm{~g} 35$ model is two times smaller than for the hotter model. To prove that our results are independent of the ambiguity in the adopted atomic parameters we performed calculations with another set of transition probabilities: the original data for Si I from Garz (1973) and the oscillator strengths for Si II lines from Blanco et al. (1995 - BBC). These results for the $8400 \mathrm{~g} 34$ model are given in the fourth line of Table 3 and demonstrate stability of the stratification analysis.

Calcium. The main indicator of the Ca stratification is the wide wings of the high-excitation Ca II lines. It is impossible to fit the cores and wings of these lines with a homogeneous abundance. The neutral calcium lines are rather insensitive to the derived stratification, and using them only can lead to a conclusion that the $\mathrm{Ca}$ distribution is homogeneous. It means that the atomic parameters for the Ca II lines play the most important role in the study of the $\mathrm{Ca}$ abundance stratification. For the two Ca II lines, 4716 and 4721, the transition probabilities from K88 and TB differ by 3 orders of magnitude, and we used the spectrum of the normal A-star HD 32115 (Bikmaev et al. 2002) to estimate the astrophysical oscillator strengths. The Ca II 4799, 5339 and 6456 lines are all very close triplets which we approximated as one line with the transition probability of the multiplet. Figure 9 shows a comparison between the observed and synthesized line profiles. The $\mathrm{Ca}$ abundance jump is not as large as in the magnetic stars 53 Cam (Babel 1992) or $\gamma$ Equ (Ryabchikova et al. 2002). In contrast to these stars, Ca is never deficient in the atmosphere of HD 204411. The size of the $\mathrm{Ca}$ abundance jump is the same for both model atmospheres of HD 204411.

Chromium. Improved wavelengths for the $\mathrm{Cr}$ I lines are taken from Murray (1992). Cr seems to be homogeneously distributed in the atmosphere of HD 204411 and it is extremely overabundant. The formal stratification solution indicates a small abundance jump $\approx 0.3-0.5 \mathrm{dex}$, but the solution is rather unstable and depends on the initial guess of the position of the abundance jump. Figure 10 shows a comparison between the 
observed and calculated line profiles. This is again different to the $\mathrm{Cr}$ distribution in magnetic stars $\gamma$ Equ and $\beta \mathrm{CrB}$ (Wade et al.2001). In the hotter atmosphere the formal abundance gradient of $\mathrm{Cr}$ changes sign.

Iron. The Fe abundance distribution was derived using numerous strong and weak Fe I and Fe II lines of different excitation. Fe is nearly solar in the upper atmosphere and by $1.5-1.7$ dex more abundant below $\log \tau_{5000}=-0.5$ to -0.7 (depending on the adopted model). Although the iron abundance jump takes place at the same optical depth as in $\gamma$ Equ, the minimum and maximum abundance in HD 204411 are by 2.4 and 0.8 dex higher than in $\gamma$ Equ. Test calculations with a different set of 20 lines give a similar iron distribution. To check the possible dependence of the derived distribution on the adopted set of oscillator strengths we calculated the Fe stratification for the $8400 \mathrm{~g} 35$ model using the Fe II data extracted only from VALD, which originally comes from the K88 list for the highexcitation lines. All stratification parameters but the Fe abundance in the upper atmosphere remain essentially the same. A decrease of the $\mathrm{Fe}$ abundance in the upper boundary by 0.36 dex leads to a decrease of the size of the abundance jump from 1.79 to 1.49 dex, but cannot cancel the stratification effects. These results provide additional support for the reliability of our stratification results. Moreover, the RU calculations for $\mathrm{Cr}$ II and Fe II were made with the same code, but we get abundance stratification for $\mathrm{Fe}$ and not for $\mathrm{Cr}$.

The abnormal strength of the high-excitation Fe II lines relative to the low-excitation lines requires concentration gradients in the deep atmospheric layers where NLTE effects are expected to be negligible. We also note that no depth-dependent microturbulent velocity models can simultaneously explain the wide wings of the strong Fe II lines and fit the normal width and increased strength of the high-excitation lines.

\section{Discussion}

Derived atmospheric parameters of HD 204411 together with the Hipparcos parallax place this star out of the MS band. Its low projected rotational velocity and weak magnetic field obviously contradict the scenario proposed by Stępień (2000) to explain the slow rotation of magnetic Ap stars. According to this hypothesis only stars with large magnetic fields become slow rotators, and they lose their angular moment before the ZAMS. There is no observational evidence for further loss of angular moment during the MS stage for normal A stars besides the usual evolutionary effects (see, for example, Hubrig et al. 2000). However, two magnetic Ap stars, CU Vir (HD 124424) and 56 Ari (HD 19832) show a retardation of their rotation. These stars are the fastest rotators among magnetic CP stars and their magnetic field is moderate, although larger than in HD 204411. CU Vir suddenly changed its rotation period, $P=0.52$ day, by only $2 \mathrm{~s}$ in 1984 (Pyper et al. 1998), and it was sufficient to result in half a period phase change ten years later. The second star, 56 Ari, $P=0.72$ day, demonstrated constant braking by 2 s over 100 years (Adelman et al. 2001). With such a rate of angular moment loss a star will be a typical slowly rotating CP star by the end of the MS lifetime. If HD 204411 was not a slow rotator originally, it might have lost its angular momentum during the MS stage in the same way as CU Vir and 56 Ari. Except for the rare-earth elements, the abundance anomalies in HD 204411 are typical for the magnetic Ap stars of Cr-type, which means that a magnetic field only slightly exceeding equipartition value $(\approx 300-400 \mathrm{G}$ for HD 204411$)$ is enough to develop the chemical peculiarities.

The existence of the vertical abundance gradients provides support for the stability of the stellar atmosphere, which seems to contradict the derived value of macroturbulence, typical for solar-like stars. Some support for the existence of the chemical separation processes in atmospheres with large-scale motions are given in the study of Landstreet (1998). He found significant atmospheric velocity fields in metallic-line (Am) stars, which were suggested to have chemical gradients in their atmospheres (Savanov \& Kochukhov 1998). HD 204411 shows only small if any overabundance of the rare-earth elements, which is one of the main peculiar characteristics of cool Ap stars with large magnetic fields. It is outside the scope of the present paper to explain the different behaviour of the abundance distributions obtained for different elements, e.g. $\mathrm{Mg}$, $\mathrm{Cr}$ and Fe. The stratification study of the Ap atmospheres is a very young field of research, and a systematic analysis of a sample of Ap stars with different rotation and magnetic fields across the whole MS band is needed to define the role of rotation, magnetic field and evolutionary effects in developing the chemical peculiarities, as well as to provide useful constraints for self-consistent theoretical diffusion calculations.

Acknowledgements. We are very grateful to A. Pamyatnykh for providing us with the evolutionary tracks. This work was supported by the FWF project $P$ 14984, by RFBR (grant 03-02-16342), Leading Scientific School grant 162.2003.02 and Presidium RAS Programme "Nonstationary phenomena in astronomy" to TR and by the Lise Meitner fellowship to OK (FWF project M757-N02).

We acknowledge important resources provided by the Vienna Atomic Line Database (VALD), the SIMBAD astronomical database and the NASA ADS.

\section{References}

Abt, H. A., \& Morrell, N. I. 1995, ApJS, 99, 135

Adelman, S. J. 1981, A\&AS, 44, 309

Adelman, S. J. 2003, A\&A, 401, 357

Adelman, S. J., Brown, B. H., Caliskan, H., Reese, D. F., \& Adelman, C. J. 1994, A\&AS, 106, 333

Adelman, S. J., Malanushenko, V., Ryabchikova, T. A., \& Savanov, I. 2001, A\&A, 375, 982

Adelman, S. J., Gulliver, A. F., Kochukhov, O. P., \& Ryabchikova, T. A. 2002, ApJ, 575, 449

Allen, M. S. 1977, ApJ, 213, 121

Anderson, E. M., Zilitis, V. A., \& Sorokina, E. S. 1967, Opt. Spectr., 23, 102

Babcock, H. W. 1949, ApJ, 110, 126

Babel, J. 1992, A\&A, 258, 449

Bard, A., \& Kock, M. 1994, A\&A, 282, 1014

Berry, H. G., Bromander, J., Curtis, L. J., \& Buchta, R. 1971, Phys. Scripta, 3, 125

Biehl, D. 1976, Ph.D. Thesis, Christian-Albrechts-Universität, Kiel, Institute für Theoretische Physik und Sternwarte 
Bikmaev, I. F., Ryabchikova, T. A., Bruntt, H., et al. 2002, A\&A, 389, 537

Black, J. H., Wisheit, J. C., \& Laviana, E. 1972, ApJ, 177, 567

Blackwell, D. E., Petford, A. D., \& Shallis, M. J. 1979, MNRAS, 186, 657

Blackwell, D. E., Petford, A. D., Shallis, M. J., \& Simmons, G. J. 1982a, MNRAS, 199, 43

Blackwell, D. E., Petford, A. D., \& Shallis, M. J. 1982b, MNRAS, 210,595

Blanco, F., Botho, B., \& Campos, J. 1995, Phys. Scr., 52, 628

Bord, D. J. 2000, A\&AS, 144, 517

Breger, M. 1976, ApJS, 32, 7

Breger, M., \& Pamyatnykh, A. 1998, A\&A, 332, 958

Buser, R., \& Kurucz, R. L. 1992, A\&A, 264, 557

Caliskan, H., \& Adelman, S. J. 1995, in Astrophysical Applications of Powerful New Databases, ed. S. J. Adelman, \& W. L. Wiese, ASP Conf. Ser., 78, 443

Cowley, C. R. 1971, Obs., 91, 139

Cowley, C. R., Elste, G. H., \& Urbanski, J. L. 1978, PASP, 90, 536

Cowley, C. R., \& Henry, R. 1979, ApJ, 233, 633

Den Hartog, E. A., Lawler, J. E., Sneden, C., \& Cowan, J. J. 2003, ApJS, 148, 543

ESA 1997, The Hipparcos and Tycho Catalogues, ESA SP-1200 (Noordjiwk: ESA)

Fitzpatrick, E. L. 1999, PASP, 111, 63

Garz, T. 1973, A\&A, 26, 471

Gerbaldi, M., Floquet, M., Faraggiana, R., \& Van't Veer-Menneret, C. 1989, A\&AS 81, 127

Ginibre, A. 1989, Phys. Scr., 39, 694

Grevesse, N., \& Sauval, A. J. 1998, Sp. Sci. Rev., 85, 161

Gurtovenko, E. A., \& Kostyk, R. I. 1981, A\&AS, 46, 239

Hauck, B., \& Mermilliod, M. 1998, A\&AS, 129, 431

Holweger, H. 2001, in Solar and Galactic Composition, ed. R. F. Wimmer-Schwengruber, AIP Conf. Proc., 598, 23

Hubrig, S., North, P., \& Medici, A. 2000, A\&A, 359, 306

Johnson, N. 2004, M.Sc. Thesis, Royal Military College of Canada

Jönsson, G., Kröll, S., Persson, A., \& Svanberg, S. 1984, Phys. Rev., A30, 2429

Kupka, F., Piskunov, N., Ryabchikova, T. A., Stempels, H. C., \& Weiss, W. W. 1999, A\&AS, 138, 119

Kupka, F., Paunzen, E., \& Maitzen, H. M. 2004, MNRAS, 352, 863

Kurucz, R. L. 1988, Trans. IAU, XXB, ed. M. McNally (Dordrecht: Kluwer), 168

Kurucz, R. L. 1993, CDROMs 13, 22, 23, SAO, Cambridge

Kurucz, R. L., \& Peytremann, E. 1975, SAO Special Report 362

Landstreet, J. D. 1998, A\&A, 338, 1041

LeBlanc, F., \& Monin, D. 2004, in IAU Symp. No. 224, The AStar Puzzle, ed. J. Zverko, W. W. Weiss, J. Žižňovský, \& S. J. Adelman, in press

Lincke, R., \& Ziegenbein, G. 1971, Z. Physik, 241, 369

Lucke, P. B. 1978, A\&A, 64, 367

Martin, G. A., Fuhr, J. R., \& Wiese, W. L. 1988, J. Phys. Chem. Ref. Data, 17, Suppl. 3

Mashonkina, L., \& Gehren, T. 2000, A\&A, 364, 249

Mathys, G. 1990, A\&A, 232, 151
May, M., Richter, J., \& Wichelmann, J. 1974, A\&AS, 18, 405

Moon, T. T., \& Dworetsky, M. M. 1985, MNRAS, 217, 305

Morgan, W. W. 1932, ApJ, 75, 46

Murray, J. E. 1992, Ph.D. Thesis, Imperial College, London

O'Brian, T. R., Wicklife, M. E., Lawler, J. E., Whaling, W., \& Brault, J. W. 1991, JOSA, B8, 1185

Palmeri, P., Quinet, P., Wyart, J.-F., \& Biémont, E. 2000, Phys. Scr., 61,323

Pamyatnykh, A. A., Dziembowski, W. A., Handler, G., \& Pikall, H. 1998, A\&A, 333, 141

Perry, C. L., Johnston, L., \& Crawford, D. L. 1982, AJ, 87, 1751

Pickering, J. C., Johansson, S., \& Smith, P. L. 2001, A\&A, 377, 361

Piskunov, N. E. 1992, in Stellar Magnetism, ed. Yu. V. Glagolevskij, \& I. I. Romanyuk (St. Petersburg: Nauka), 92

Piskunov, N., \& Kupka, F. 2001, ApJ, 547, 1040

Preston, G. W. 1970, PASP, 82, 878

Preston, G. W. 1971, ApJ, 164, 309

Przybilla, N., Butler, K., Becker, S. R., Kudritzki, R. P., \& Venn, K. A. 2000, A\&A, 359,1085

Przybilla, N., Butler, K., Becker, S. R., \& Kudritzki, R. P. 2001, A\&A, 369,1009

Pyper, D. M., Ryabchikova, T., Malanushenko, V., et al. 1998, A\&A, 339,822

Raassen, A. J. J., \& Uylings, P. H. M. 1998, A\&A, 340, 300

Roby, S. W., \& Lambert, D. L. 1990, ApJS, 73, 67

Royer, F., Grenier, S., Baylac, M.-O., Gómez, A. E., \& Zorec, J. 2002, A\&A, 393, 897

Ryabchikova, T. A., Piskunov, N., Stempels, H. C., Kupka, F., \& Weiss, W. W. 1999, Phys. Scr., T83, 162

Ryabchikova, T., Piskunov, N., Kochukhov, O., et al. 2002, A\&A, 384, 545

Ryabchikova, T., Wade, G., \& LeBlanc, F. 2003, in IAU Symp., No. 210, Modelling of Stellar Atmospheres, ed. N. E. Piskunov, W. W. Weiss, \& D. F. Gray, ASP, 301

Ryabchikova, T., Nesvacil, N., Weiss, W. W., Kochukhov, O., \& Stütz, C. 2004, A\&A, 423, 705

Sargent, W. L. W., Strom, K. M., \& Strom, S. E. 1969, ApJ, 157, 1265

Savanov, I. S., \& Kochukhov, O. P. 1998, Astron. Lett., 24, 516

Schulz-Gulde, E. 1969, JQSRT, 9, 13

Seaton, M. J., Zeippen, C. J., Tully, J. A., et al. 1992, Rev. Mex. A\&A, 23, 19

Smirnov, O. M., \& Ryabchikova, T. A. 1995, Astron. Rep., 39, 755

Smith, G., \& Raggett, D. St. J. 1981, J. Phys. B: At. Mol. Phys., 14, 4015

Stępień, K. 2000, A\&A, 353, 227

Stift, M. J. 2000, A Pecul. Neslett, 33

Stift, M. J., \& Leone, F. 2003, A\&A, 398, 411

Takeda, Y. 1997, PASJ, 49, 471

Tody, D. 1986, The IRAF Data Reduction and Analysis System, in Proc. SPIE Instrumentation in Astronomy VI, ed. D. L. Crawford, 627, 733

Wade, G. A., Ryabchikova, T. A., Bagnulo, S., \& Piskunov, N. 2001, in Magnetic fields across the Hertzprung-Russel diagram, ed. G. Mathys, S. K. Solanki, \& T. Wickramasinghe, ASP Conf. Ser., 248,341 